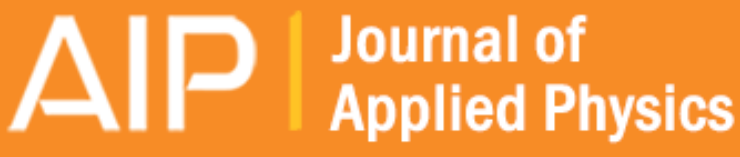

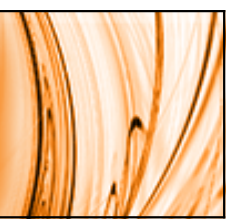

\section{Multiferroic tunnel junctions and ferroelectric control of magnetic state at interface (invited)}

Y. W. Yin, M. Raju, W. J. Hu, J. D. Burton, Y.-M. Kim, A. Y. Borisevich, S. J. Pennycook, S. M. Yang, T. W. Noh,

A. Gruverman, X. G. Li, Z. D. Zhang, E. Y. Tsymbal, and Qi Li

Citation: Journal of Applied Physics 117, 172601 (2015); doi: 10.1063/1.4913753

View online: http://dx.doi.org/10.1063/1.4913753

View Table of Contents: http://scitation.aip.org/content/aip/journal/jap/117/17?ver=pdfcov

Published by the AIP Publishing

\section{Articles you may be interested in}

Prediction of giant magnetoelectric effect in LaMnO3/BaTiO3/SrMnO3 superlattice: The role of n-type

$\mathrm{SrMnO} / \mathrm{LaMnO} 3$ interface

J. Appl. Phys. 116, 074102 (2014); 10.1063/1.4893370

Interface-modification-enhanced tunnel electroresistance in multiferroic tunnel junctions

J. Appl. Phys. 116, 053703 (2014); 10.1063/1.4892592

Coexistence of four resistance states and exchange bias in La0.6Sr0.4MnO3/BiFeO3/La0.6Sr0.4MnO3 multiferroic tunnel junction

Appl. Phys. Lett. 104, 043507 (2014); 10.1063/1.4863741

Prediction of ferroelectric stability and magnetoelectric effect of asymmetric multiferroic tunnel junctions Appl. Phys. Lett. 102, 152906 (2013); 10.1063/1.4801306

Coexistence of tunneling magnetoresistance and electroresistance at room temperature in $\mathrm{La} 0.7 \mathrm{Sr} 0.3 \mathrm{MnO} /(\mathrm{Ba}$, $\mathrm{Sr}) \mathrm{TiO} 3 / \mathrm{La} 0.7 \mathrm{Sr} 0.3 \mathrm{MnO} 3$ multiferroic tunnel junctions

J. Appl. Phys. 109, $07 D 915$ (2011); 10.1063/1.3564970

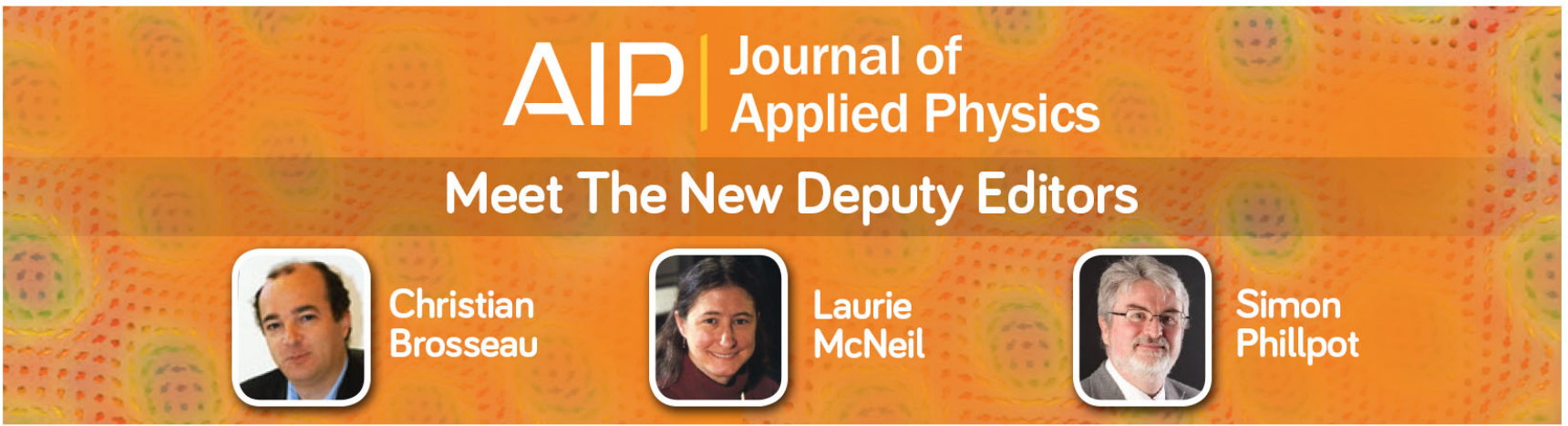




\title{
Multiferroic tunnel junctions and ferroelectric control of magnetic state at interface (invited)
}

\author{
Y. W. Yin, ${ }^{1}$ M. Raju, ${ }^{1}$ W. J. Hu, ${ }^{2}$ J. D. Burton, ${ }^{3}$ Y.-M. Kim, ${ }^{4}$ A. Y. Borisevich, ${ }^{4}$ \\ S. J. Pennycook, ${ }^{4}$ S. M. Yang, ${ }^{5}$ T. W. Noh, ${ }^{5}$ A. Gruverman, ${ }^{3}$ X. G. Li, ${ }^{6}$ Z. D. Zhang, ${ }^{7}$ \\ E. Y. Tsymbal, ${ }^{3}$ and Qi $\mathrm{Li}^{1, \mathrm{a})}$ \\ ${ }^{1}$ Department of Physics, Pennsylvania State University, University Park, Pennsylvania 16802, USA \\ ${ }^{2}$ Physical Sciences and Engineering Division, King Abdullah University of Science and Technology, \\ Thuwal 23955-6900, Saudi Arabia \\ ${ }^{3}$ Department of Physics and Astronomy and Nebraska Center for Materials and Nanoscience, \\ University of Nebraska, Lincoln, Nebraska 68588-0299, USA \\ ${ }^{4}$ Materials Science and Technology Division, Oak Ridge National Laboratory, Oak Ridge, Tennessee 37831, \\ USA \\ ${ }^{5}$ IBS-Center for Functional Interfaces of Correlated Electron Systems, Department of Physics and Astronomy, \\ Seoul National University, Seoul 151-747, South Korea \\ ${ }^{6}$ Hefei National Laboratory for Physical Sciences at Microscale, Department of Physics, University of Science \\ and Technology of China, Hefei 230026, People's Republic of China \\ ${ }^{7}$ Shenyang National Laboratory for Materials Science, Institute of Metal Research, \\ Chinese Academy of Sciences, Shenyang 110016, China
}

(Presented 4 November 2014; received 22 September 2014; accepted 12 October 2014; published online 3 March 2015)

\begin{abstract}
As semiconductor devices reach ever smaller dimensions, the challenge of power dissipation and quantum effect place a serious limit on the future device scaling. Recently, a multiferroic tunnel junction (MFTJ) with a ferroelectric barrier sandwiched between two ferromagnetic electrodes has drawn enormous interest due to its potential applications not only in multi-level data storage but also in electric field controlled spintronics and nanoferronics. Here, we present our investigations on four-level resistance states, giant tunneling electroresistance (TER) due to interfacial magnetoelectric coupling, and ferroelectric control of spin polarized tunneling in MFTJs. Coexistence of large tunneling magnetoresistance and TER has been observed in manganite/( $\mathrm{Ba}, \mathrm{Sr}) \mathrm{TiO}_{3} / \mathrm{manga}^{-}$ nite MFTJs at low temperatures and room temperature four-resistance state devices were also obtained. To enhance the TER for potential logic operation with a magnetic memory, $\mathrm{La}_{0.7} \mathrm{Sr}_{0.3} \mathrm{MnO}_{3} / \mathrm{BaTiO}_{3} / \mathrm{La}_{0.5} \mathrm{Ca}_{0.5} \mathrm{MnO}_{3} / \mathrm{La}_{0.7} \mathrm{Sr}_{0.3} \mathrm{MnO}_{3}$ MFTJs were designed by utilizing a bilayer tunneling barrier in which $\mathrm{BaTiO}_{3}$ is ferroelectric and $\mathrm{La}_{0.5} \mathrm{Ca}_{0.5} \mathrm{MnO}_{3}$ is close to ferromagnetic metal to antiferromagnetic insulator phase transition. The phase transition occurs when the ferroelectric polarization is reversed, resulting in an increase of TER by two orders of magnitude. Tunneling magnetoresistance can also be controlled by the ferroelectric polarization reversal, indicating strong magnetoelectric coupling at the interface. (C) 2015 AIP Publishing LLC.
\end{abstract}

[http://dx.doi.org/10.1063/1.4913753]

\section{INTRODUCTION}

As the demands for faster, smaller, and non-volatile electronics increase, traditional silicon based semiconductor devices have been pushed to ever smaller dimensions. However, power dissipation and finite size effect have posted constraint on device miniaturization. Several new concepts for the next generation devices for information processing and storage have been proposed and studied recently. ${ }^{1-3}$ Multiferroic tunnel junctions (MFTJs), employing a ferroelectric tunnel barrier layer in a magnetic tunnel junction (MTJ), have become one of the very promising approaches to the new generation of multifunctional devices. ${ }^{4,5}$ From the MFTJ constituents, it is an MTJ $^{6-8}$ with a ferroelectric barrier or a ferroelectric tunnel junction (FTJ $)^{9-12}$ with two ferromagnetic (FM) electrodes (see Fig. 1(a) for the schematic views of MTJ, FTJ, and

\footnotetext{
a) Author to whom correspondence should be addressed. Electronic mail: Qil1@psu.edu.
}

MFTJ). As an MTJ, the tunneling current of an MFTJ depends on the relative orientation of the magnetization of the two ferromagnetic electrodes, which can be changed between parallel and antiparallel magnetic states by an applied magnetic field. ${ }^{6-8}$ This is known as tunneling magnetoresistance (TMR) effect. While as an FTJ, an applied electric field can reverse the ferroelectric polarization of the barrier in an MFTJ, which will affect the junction resistance by changing the electrostatic potential or interface bonding strength (thus the density of states). ${ }^{4,5,9-12}$ For instance, if the two electrodes of an FTJ have different screening lengths, the electrostatic effect resulting from incomplete screening of the polarization charges at the interface will make the potential energy profile unsymmetrical. This will lead to different average energy barriers for different barrier polarization directions (shown in Fig. 1(b)). ${ }^{12}$ From the quantum tunneling model, this will result in different currents and therefore a tunneling electroresistance (TER) effect. It should be noted that the TER effect in an FTJ is 
(a)

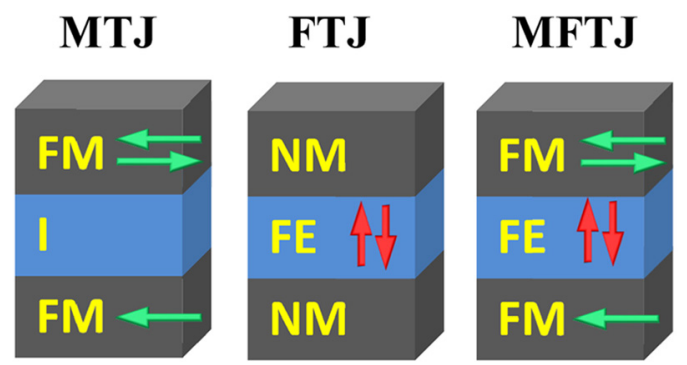

(b)

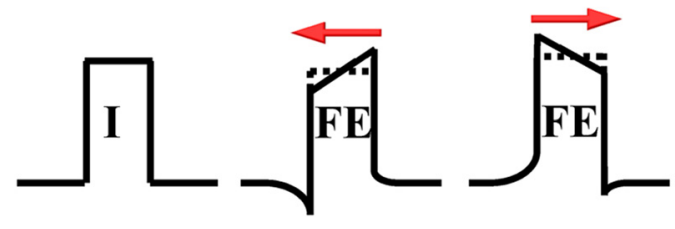

FIG. 1. (a) Schematic view of the MTJ, FTJ, and MFTJ. (b) Schematic representation of the potential energy profile in a junction with a normal dielectric insulator barrier (I) and ferroelectric (FE) barriers for polarization pointing to the left and right, assuming screening length at left side is larger than right side. FM, FE, normal metal (NM), and insulating (I) layers are indicated in the proper positions. Green and red arrows indicate orientations of magnetization and ferroelectric polarization, respectively.

linked to the ferroelectric polarization direction and hence is distinguished from other resistive switching mechanisms such as the interfacial electrochemical modification or conducting filament formation which can also be observed in oxide materials. ${ }^{13,14}$ One of the key properties of an MFTJ is the coexistence of the TMR and TER effects. Since both have two resistance states, an MFTJ will be a four-state resistance device, in which resistance can be switched among these states by external electric and magnetic fields. This provides a new approach to achieve high density memories. Furthermore, the information coded in an MFTJ through the different magnetization configurations in the electrodes and ferroelectricity orientations in the barrier are non-volatile. Due to these advantages, experimental efforts have been made and evidences of the four states have been demonstrated by several groups in MFTJs with $(\mathrm{Ba}, \mathrm{Sr}) \mathrm{TiO}_{3}, \mathrm{BiFeO}_{3}$, or $\mathrm{PbZr}_{0.2} \mathrm{Ti}_{0.8} \mathrm{O}_{3}$ ferroelectric tunnel barriers. ${ }^{15-20}$ Very recently, the realization of an epitaxial perovskite $\mathrm{BaTiO}_{3}$ (BTO) based FTJ on silicon suggests the possibility of integrating FTJs/ MFTJs on silicon wafers, thus the integration with semiconductor electronics. $^{21}$

In addition to the capability to control electron and spin tunneling via ferromagnetic and ferroelectric polarizations in the electrode and barrier layers, the MFTJs have also been predicted to have other advantages beyond the simple addition of an MTJ with an FTJ. In an MFTJ, the carrier concentration and/or chemical bonding strength manipulations at the ferroelectric/ferromagnetic interfaces may give rise to an interfacial magnetoelectric effect, which can change the magnetic anisotropy, coercivity, or even the interfacial magnetic structure by an electric field through switchable ferroelectric polarization. ${ }^{22-25}$ This strong interfacial magnetoelectric coupling in MFTJs provides an alternative energy-efficient route to manipulate spins by an electric field besides the spin transfer torque ${ }^{26}$ and electric field assisted switching effects ${ }^{27}$ in MTJs. Meanwhile, the interrelationship between ferroelectricity of the barrier layer and ferromagnetism of the electrodes through an interface magnetoelectric effect also affects the functional properties of TMR and TER in MFTJs. ${ }^{17,28}$ Hence the interface magnetoelectric coupling effect provides a playground to design MFTJs with better performance. $^{29,30}$

Since large TER effect is very desirable for utilizing the device as a resistance switch or for signal processing, several methods have been proposed or tested to improve the TER ratio in an FTJ or MFTJ. ${ }^{9}$ For example, special barrier/electrode interfacial designs have been used to improve TER, such as inserting a thin nonpolar dielectric layer at the interface between the ferroelectric barrier and the metal electrode, ${ }^{31}$ inserting a thin magnetic phase transition layer at the interface, ${ }^{29}$ using $\mathrm{Nb}$-doped $\mathrm{SrTiO}_{3}$ substrate as a semiconducting bottom electrode, ${ }^{32}$ or changing the $\mathrm{La}_{1-x} \mathrm{~A}_{x} \mathrm{MnO}_{3}$ $(\mathrm{A}=\mathrm{Sr}, \mathrm{Ca})$ electrode composition to the phase boundary at $x=0.2$ and $0.5{ }^{30}$ In addition, in thick barrier case when the thermionic injection transport dominates, an increase of TER has also been predicted. ${ }^{33}$

In this paper, we present the four resistance state effect, enhancement of TER using interfacial magnetoelectric coupling, and ferroelectric control of the TMR in MFTJs. After the Introduction section, Sec. II describes the large TMR and TER effects in $\mathrm{La}_{0.7} \mathrm{Ca}_{0.3} \mathrm{MnO}_{3}$ (LCMO)/ $/ \mathrm{Ba}_{0.5} \mathrm{Sr}_{0.5} \mathrm{TiO}_{3} /$ LCMO MFTJs at low temperatures, and the room temperature four-state effect obtained in $\mathrm{La}_{0.7} \mathrm{Sr}_{0.3} \mathrm{MnO}_{3}$ (LSMO)/ $\mathrm{Ba}_{0.95} \mathrm{Sr}_{0.05} \mathrm{TiO}_{3} / \mathrm{LSMO}$ MFTJs. In Sec. III, we have designed and fabricated LSMO/BTO/La ${ }_{0.5} \mathrm{Ca}_{0.5} \mathrm{MnO}_{3} / \mathrm{LSMO}$ MFTJs with an ultrathin $\mathrm{La}_{0.5} \mathrm{Ca}_{0.5} \mathrm{MnO}_{3}$ film inserted between the LSMO ferromagnetic electrode and the BTO ferroelectric barrier, in which a giant and reproducible TER effect was obtained with a reversal of ferroelectric polarization. In Sec. IV, we show the control of TMR, thus the spin polarization, with the ferroelectric polarization reversal.

\section{FOUR RESISTANCE STATES IN MFTJS}

Thin insulating barriers with persistent ferroelectricity, asymmetric interfaces, and high spin polarization are critical for four-state effect in MFTJs. We chose (Ba, $\mathrm{Sr}) \mathrm{TiO}_{3}$ as ferroelectric barrier layer in which the ferroelectricity can be persistent down to one unit cell (uc) under proper strain condition $^{34}$ and half-metallic LCMO and LSMO as ferromagnetic electrodes for the proper lattice match with the barrier. Fig. 2(a) shows a schematic diagram of the planner junction, and Fig. 2(b) depicts the optical microscope image of a completed $\mathrm{LCMO} / \mathrm{Ba}_{0.5} \mathrm{Sr}_{0.5} \mathrm{TiO}_{3} / \mathrm{LCMO} \mathrm{MFTJ}$ at the junction area. The junctions were made using a standard planar trilayer tunnel junction structure, compatible with the standard processing procedure for semiconductor devices. Epitaxial $\mathrm{LCMO} / \mathrm{Ba}_{0.5} \mathrm{Sr}_{0.5} \mathrm{TiO}_{3} / \mathrm{LCMO}$ trilayers were made by pulsedlaser deposition followed by photolithography and ion milling. $\mathrm{MgO}$ or $\mathrm{SiO}_{2}$ layer was sputtered in between the top gold and bottom LCMO leads to isolate the contacts. ${ }^{29}$

Figure 2(c) shows the four-resistance-state for an LCMO/ $\mathrm{Ba}_{0.5} \mathrm{Sr}_{0.5} \mathrm{TiO}_{3} / \mathrm{LCMO}$ MFTJ with an area of $8 \times 8 \mu \mathrm{m}^{2}$ and the nominal barrier thickness of $\sim 1.2 \mathrm{~nm}$ (3 uc). In Fig. 2(c), the $R$ - $H$ loops for both polarization states show high-quality standard MTJ behaviors, and the TMR ratio is about $180 \%$ 

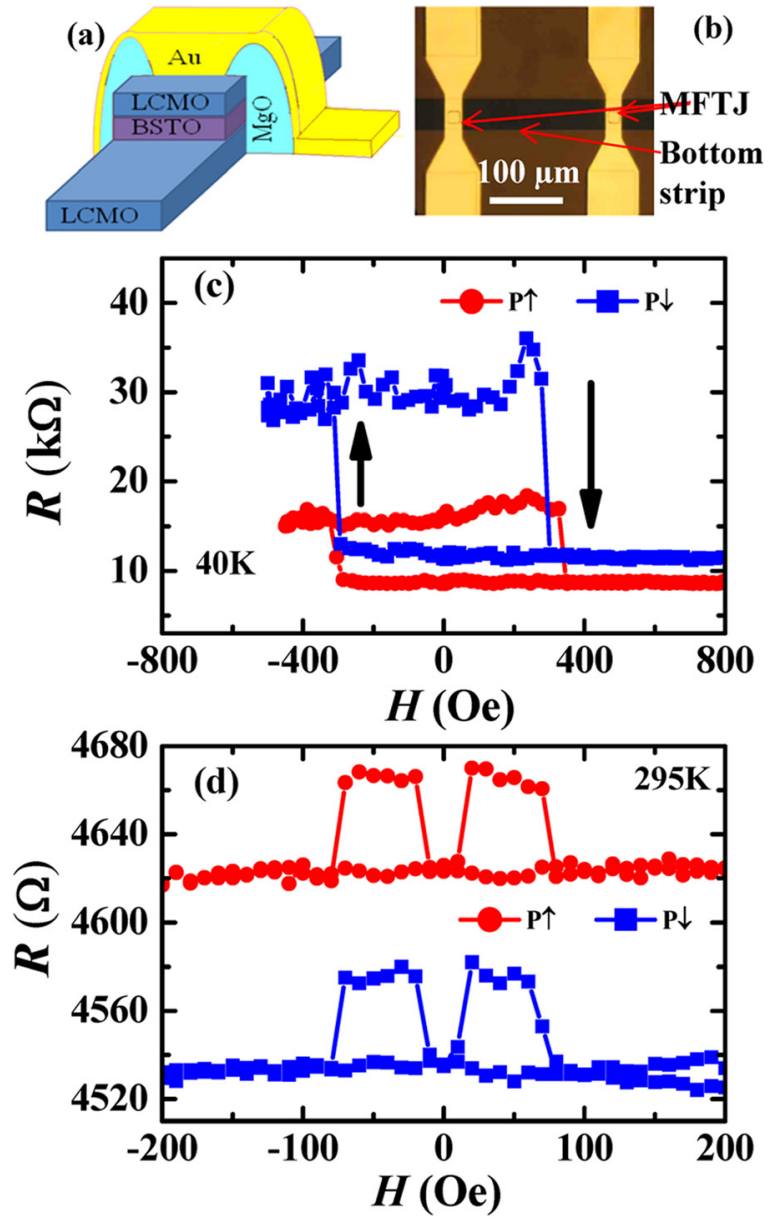

FIG. 2. (a) Schematic drawing of the MFTJ sample structure. (b) Picture of a completed $\mathrm{LCMO} / \mathrm{Ba}_{0.5} \mathrm{Sr}_{0.5} \mathrm{TiO}_{3} / \mathrm{LCMO}$ MFTJ at the junction area. (c) Junction resistance $v s$. magnetic field loop at a bias of $0.1 \mathrm{~V}$ and temperature of $40 \mathrm{~K}$ for an $\mathrm{LCMO} / \mathrm{Ba}_{0.5} \mathrm{Sr}_{0.5} \mathrm{TiO}_{3} / \mathrm{LCMO} \mathrm{MFTJ}$ with the ferroelectric polarization poled downward and upward. The arrows indicate the direction of field sweeping sequence. (b) Resistance vs. magnetic field curves for an LSMO/ $\mathrm{Ba}_{0.95} \mathrm{Sr}_{0.05} \mathrm{TiO}_{3} / \mathrm{LSMO}$ MFTJ for the two ferroelectric polarization directions at room temperature.

and $100 \%$ for the down and up polarization states. At lower temperatures, the TMR can reach $300 \%$ at $4.2 \mathrm{~K}$. By applying a pulse voltage to switch the ferroelectric orientation between the down and up polarization states, the $R-H$ loop switches between the two $R-H$ curves. At zero magnetic and electric fields, the device has four distinct resistance states, corresponding to the combination of magnetic parallel and antiparallel states and ferroelectric polarization down and up states. Since the same electrode materials are used, the TER effect is related to the differences in the atomic arrangement between the top and bottom interfaces of the barrier, similar to the theoretical calculation on all-oxide $\mathrm{SrRuO}_{3} / \mathrm{BTO} / \mathrm{SrRuO}_{3}$ MFTJ. $^{28}$ The TER ratios are $\sim 90 \%$ and $\sim 30 \%$ for the antiparallel and parallel magnetic states, respectively. These values are comparable to the currently used TMR memories and therefore can potentially be used as a combination memory with doubling the memory states of a MTJ.

As we know, both the ferromagnetic transition temperatures of the LCMO bottom layer and the ferroelectric Curie temperature of the $\mathrm{Ba}_{0.5} \mathrm{Sr}_{0.5} \mathrm{TiO}_{3}$ barrier layer are below room temperature $(250-270 \mathrm{~K})$. Therefore, the LCMO/
$\mathrm{Ba}_{0.5} \mathrm{Sr}_{0.5} \mathrm{TiO}_{3} / \mathrm{LCMO}$ MFTJ cannot work at room temperature. To overcome this problem, LSMO/ $\mathrm{Ba}_{0.95} \mathrm{Sr}_{0.05} \mathrm{TiO}_{3} /$ LSMO MFTJs were fabricated. The LSMO layers have a magnetic Curie temperature $\sim 350 \mathrm{~K}$ and $\mathrm{Ba}_{0.95} \mathrm{Sr}_{0.05} \mathrm{TiO}_{3}$ has a ferroelectric Curie point $\sim 390 \mathrm{~K} .{ }^{15}$ Fig. $2(\mathrm{~d})$ shows the $R-H$ curves at room temperature for an $\mathrm{LSMO} / \mathrm{Ba}_{0.95} \mathrm{Sr}_{0.05} \mathrm{TiO}_{3} /$ LSMO MFTJ with the size of $10 \times 20 \mu \mathrm{m}^{2}$ and the nominal barrier thickness of $\sim 3.5 \mathrm{~nm}$. The four resistance states are observed at room temperature. Note although room temperature four resistance states can be observed in LSMO/ $\mathrm{Ba}_{0.95} \mathrm{Sr}_{0.05} \mathrm{TiO}_{3} / \mathrm{LSMO}$ MFTJs, the TER and TMR ratios are fairly small in comparison with the previous devices. Further studies and selection of higher $\mathrm{T}_{\mathrm{c}}$ materials to improve the performance of MFTJ at room temperature are needed for potential applications.

\section{ENHANCEMENT OF TER THROUGH AN INTERFACIAL FERROELECTRIC INDUCED PHASE TRANSITION}

Recently, using first-principles density-functional calculations, it was predicted that in an $\mathrm{La}_{1-x} \mathrm{Sr}_{x} \mathrm{MnO}_{3} / \mathrm{BTO} /$ $\mathrm{La}_{1-x} \mathrm{Sr}_{x} \mathrm{MnO}_{3}$ MFTJ, the magnetic order of the interface layer in $\mathrm{La}_{1-x} \mathrm{Sr}_{x} \mathrm{MnO}_{3}$ adjacent to $\mathrm{BaTiO}_{3}$ would be changed across the phase boundary $x \sim 0.5$ between a ferromagnetic metallic phase $(x<0.5)$ and A-type antiferromagnetic insulating phase $(x>0.5)$ by the ferroelectric switching. The tunneling current will then be changed significantly due to a spin valve effect, resulting in a much larger TER than that of a normal FTJ. ${ }^{25}$ Experimentally, we have designed a bilayer tunneling barrier in LSMO/BTO/La ${ }_{0.5} \mathrm{Ca}_{0.5} \mathrm{MnO}_{3} / \mathrm{LSMO}$ MFTJs, in which one layer is a ferroelectric BTO and the other $\mathrm{La}_{0.5} \mathrm{Ca}_{0.5} \mathrm{MnO}_{3}$ is close to ferromagnetic metal to antiferromagnetic insulator phase transition. ${ }^{29}$ The mechanism of the $T E R$ enhancement in this structure is schematically shown in Figs. 3(a) and 3(b). For the polarization to the upward direction, pointing to the thin $\mathrm{La}_{0.5} \mathrm{Ca}_{0.5} \mathrm{MnO}_{3}$ layer (see Fig. 3(a)), the screening electron accumulation or hole depletion will reduce the doping level $x$ of $\mathrm{La}_{0.5} \mathrm{Ca}_{0.5} \mathrm{MnO}_{3}$ to $x<0.5$ side, which is in ferromagnetic metallic phase. ${ }^{35}$ While at the other side of BTO barrier, because the stoichiometry of LSMO is far enough away from the phase boundary, the theoretical calculation demonstrated that the magnetic reconstruction will not occur. ${ }^{36}$ On the other hand, for the polarization to the

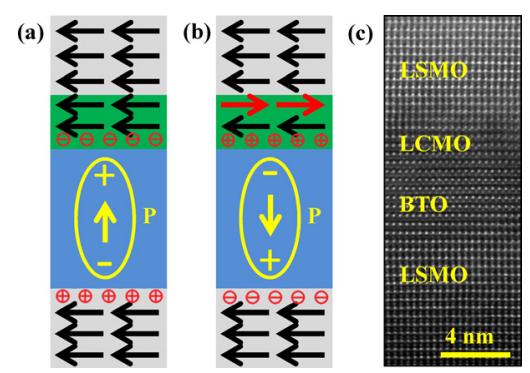

FIG. 3. (a) and (b) Schematic demonstration of the screening charge accumulation in the electrodes for the $\mathrm{LSMO} / \mathrm{BTO} / \mathrm{La}_{0.5} \mathrm{Ca}_{0.5} \mathrm{MnO}_{3} / \mathrm{LSMO}$ MFTJ with ferroelectric polarization (a) upwards and (b) downwards. (c) The cross-sectional HRTEM image of an as-grown LSMO/BTO/ $\mathrm{La}_{0.5} \mathrm{Ca}_{0.5} \mathrm{MnO}_{3} / \mathrm{LSMO}$ multilayer at the interfacial region. The arrows indicate the magnetic moments on the Mn sites. 
downward direction, pointing away from the $\mathrm{La}_{0.5} \mathrm{Ca}_{0.5} \mathrm{MnO}_{3}$ film (see Fig. 3(b)), the electron depletion or hole accumulation will change the $\mathrm{La}_{0.5} \mathrm{Ca}_{0.5} \mathrm{MnO}_{3}$ to $x>0.5$ side and push it into antiferromagnetic insulating phase. ${ }^{35}$ The change of conductance in $\mathrm{La}_{0.5} \mathrm{Ca}_{0.5} \mathrm{MnO}_{3}$ along [001] direction will effectively increase the barrier thickness and reduce the tunneling current. More importantly, a few unit-cells of antiferromagnetic $\mathrm{La}_{0.5} \mathrm{Ca}_{0.5} \mathrm{MnO}_{3}$ will act as an atomic-scale spin valve by filtering spin-dependent current. Thus, a much larger $T E R$ effect can be expected.

Similar to the normal trilayer MFTJs discussed in Part II, the LSMO/BTO/La ${ }_{0.5} \mathrm{Ca}_{0.5} \mathrm{MnO}_{3} / \mathrm{LSMO}$ MFTJs were also grown epitaxially on $\mathrm{SrTiO}_{3}(001)$ substrates by pulsedlaser deposition, and the structural characterization of duplicate heterostructures fabricated under identical conditions were performed by HRTEM, as shown in Fig. 3(c). Fig. 4(a) shows the resistance memory loops as a function of pulsed poling voltage at $40 \mathrm{~K}$ for an MFTJ with an area of $15 \times 15 \mu \mathrm{m}^{2}$ and with $2 \mathrm{uc} \mathrm{La}_{0.5} \mathrm{Ca}_{0.5} \mathrm{MnO}_{3}$ inserted between the $3 \mathrm{~nm} \mathrm{BaTiO}$ barrier and top LSMO electrode (sample ID: $J 1-2 u c, 2 u c$ denotes the thickness of $\mathrm{La}_{0.5} \mathrm{Ca}_{0.5} \mathrm{MnO}_{3}$ layer). Each data point in the hysteresis curve was measured at a fixed voltage bias $10 \mathrm{mV}$ after applying a $\sim 20 \mathrm{~ms}$ voltage pulse. As expected, the resistance is higher when large enough positive voltage was applied to polarize the ferroelectricity downward, and will switch to a lower resistance state when the ferroelectric polarization is reversed by a large enough negative voltage. The negative voltage will switch the ferroelectric polarization upward, pointing to the $\mathrm{La}_{0.5} \mathrm{Ca}_{0.5} \mathrm{MnO}_{3}$, which will absorb the hole depletion. This will push the $\mathrm{La}_{0.5} \mathrm{Ca}_{0.5} \mathrm{MnO}_{3}$ into a more ferromagnetic metallic phase, reduce the barrier width with no spin filter effect at the interface, and thus lead to a huge resistance drop. The $T E R$ ratio reaches $\sim 5,000 \%$ at $40 \mathrm{~K}$.

For comparison, the $R-V_{\text {pulse }}$ loop of a normal LSMO/ BTO/LSMO trilayer MFTJ without the inserted $\mathrm{La}_{0.5} \mathrm{Ca}_{0.5} \mathrm{MnO}_{3}$ layer (area $12 \times 12 \mu \mathrm{m}^{2}$ and barrier $4 \mathrm{~nm}$ ) prepared with the same condition (sample n: $J 2$ ), was also measured and shown in Fig. 4(b). The TER ratio for this junction is only $~ 30 \%$, two orders of magnitudes smaller than that of $J 1-2 u c$ in Fig. 4(a). This clearly indicates the decisive role of the $\mathrm{La}_{0.5} \mathrm{Ca}_{0.5} \mathrm{MnO}_{3}$ interlayer on the TER magnitude. Besides the large magnitude of $T E R$, the reproducibility and the

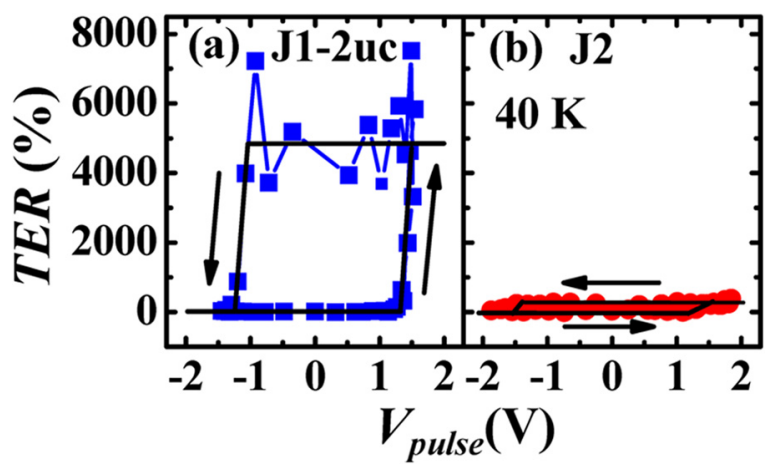

FIG. 4. The resistance memory loops as a function of pulsed poling voltage at $40 \mathrm{~K}$ for (a) junction $J 1-2 u c$ and (b) $J 2$. The solid lines are guide to the eyes. The arrows indicate the direction of pulse sequence.

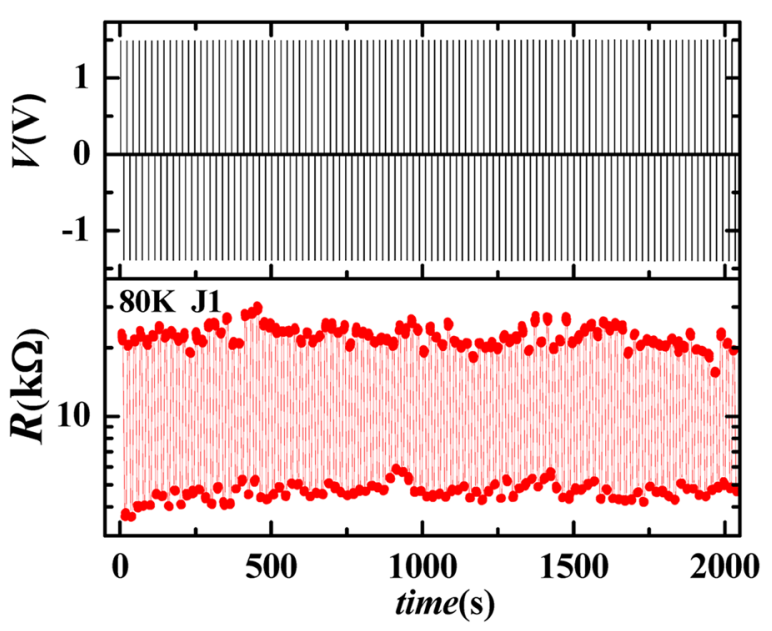

FIG. 5. Resistance switching between positive and negative polarization states at $80 \mathrm{~K}$ (bottom panel) in response to applied voltage pulses (top panel).

robustness of the two states are other decisive characteristics of an MFTJ for potential applications. Fig. 5 shows the resistance switching measurement at $80 \mathrm{~K}$ for $J 1-2 u c$. By a series of consecutive switching of the barrier polarization (voltage pulses $\pm 1.4 \mathrm{~V}$ ), the resistance switches back and forth between two resistance states up to 100 times with no indication of deterioration.

To exclude other resistance switching mechanisms, ${ }^{13,14}$ piezoresponse force microscopy (PFM) has been carried out to show that the ferroelectric polarization reversal is the underlying mechanism for the TER resistive switching in the samples. ${ }^{29}$ Fig. 6 shows the PFM phase and amplitude hysteresis loops for an $\mathrm{LSMO} / \mathrm{BTO}(3 \mathrm{~nm}) / \mathrm{La}_{0.5} \mathrm{Ca}_{0.5} \mathrm{MnO}_{3} / \mathrm{LSMO}$ MFTJ. It is clearly shown that when the polarization direction of the ferroelectric BTO layer reverses, the phase alters nearly by $180^{\circ}$. As shown in the inset of Fig. 6 , the coercive voltages obtained from PFM measurements are consistent with the threshold switching voltages obtained from the TER measurement in a wide range of temperatures, indicating that the ferroelectric polarization reversal is the underlying mechanism for the resistive switching of our junctions. ${ }^{14,29}$

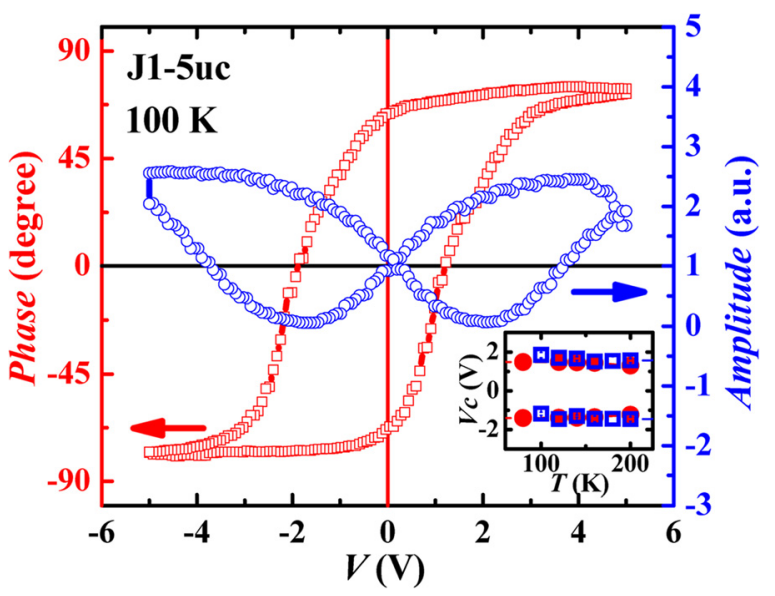

FIG. 6. Main panel: PFM phase (red curve) and amplitude (blue) hysteresis loops for a $\mathrm{LSMO} / \mathrm{BTO}(3 \mathrm{~nm}) / \mathrm{La}_{0.5} \mathrm{Ca}_{0.5} \mathrm{MnO}_{3} / \mathrm{LSMO}$ MFTJ. The inset shows the coercive voltage as a function of temperature determined from the PFM amplitude (blue symbols) and the threshold switching voltage obtained from the TER measurements (red symbols). 
To obtain the barrier information of the MFTJ, the $I-V$ curves for $J 1-2 u c$ and $J 2$ at different temperatures were plotted and fitted by the trapezoidal barrier tunneling model. ${ }^{37}$ Figs. 7 (a) and 7(b) show the representative $I$ - $V$ curves of $J 1-2 u c$ and $J 2$ measured at $80 \mathrm{~K}$ after the ferroelectricity poled upwards (blue) and downwards (red). The fitted average barrier height/width at $80 \mathrm{~K}$ in upward and downward states are $0.20 \mathrm{eV} / 3.5 \mathrm{~nm}$ and $0.12 \mathrm{eV} / 5.2 \mathrm{~nm}$ for $J 1-2 u c$, and $0.24 \mathrm{eV} / 2.7 \mathrm{~nm}$ and $0.22 \mathrm{eV} / 2.9 \mathrm{~nm}$ for $J 2$, which fitting errors are $<1 \%$. The significant difference between the $I-V$ curves measured at different polarization states for $J 1-2 u c$ and $J 2$ suggests the strong role of $\mathrm{La}_{0.5} \mathrm{Ca}_{0.5} \mathrm{MnO}_{3}$ interface layer in improving the TER effect. For junction $J 1-2 u c$, the fitting results indicate that when the resistance is changed from the low to high resistance state upon the ferroelectric polarization reversal, the effective barrier width is increased by about $1.5 \mathrm{~nm}$. This change in the barrier width for the two resistance states is consistent with the phase transition model for $\mathrm{La}_{0.5} \mathrm{Ca}_{0.5} \mathrm{MnO}_{3}$ with ferroelectric reversal.

We can calculate the TER ratio from the $I-V$ curves, as shown in Fig. 8(a). The TER ratio increases with decreasing bias and reaches up to $\sim 10000 \%$ for $J 1-2 u c$ at $5 \mathrm{~K}$, while only $\sim 30 \%$ for $\mathrm{J} 2$. The TER ratio increases with decreasing temperature, especially for $J 1-2 u c .^{29}$ To compare the bias dependent trend for the TER, the normalized TER/TER $(0 \mathrm{~V})$ for $J 1-2 u c$ and $J 2$ at $5 \mathrm{~K}$ and $80 \mathrm{~K}$ was plotted in Fig. 8(b). It can be seen that the bias dependence is more significant for sample $J 1-2 u c$ at lower temperatures. This can be explained by a thermally activated inelastic conductance channel through chains of localized states in the barrier, which has been found for tunnel junctions with $\mathrm{SrTiO}_{3}$ and $\mathrm{MgO}$ barriers. ${ }^{38,39}$ The fitting of the temperature dependence of the junction resistance also agrees with this model. ${ }^{29}$ This conduction mechanism is strongly temperature, bias, and barrier thickness dependent

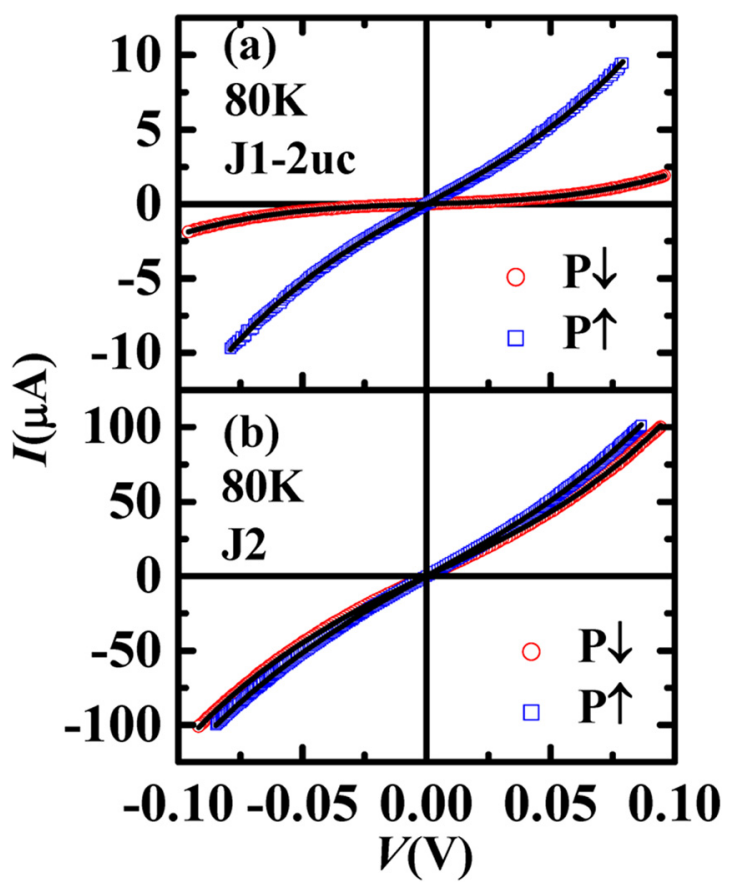

FIG. 7. $I$ - $V$ curves for (a) $J 1-2 u c$ and (b) $J 2$ at $80 \mathrm{~K}$ for opposite polarization directions. Black solid lines are the fitting results.
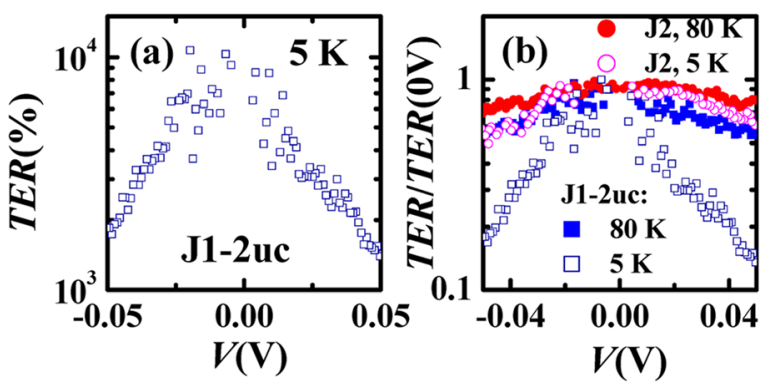

FIG. 8. (a) Bias dependence of TER for $J 1-2 u c$ at $5 \mathrm{~K}$. (b) normalized TER for $J 1-2 u c$ and $J 2$ at $80 \mathrm{~K}$ and $5 \mathrm{~K}$.

and contributes more to the transport with increasing temperature and bias. ${ }^{40}$ Unlike direct tunneling, the defect-mediated inelastic tunneling is less sensitive to polarization orientation and will reduce TER. This explains why the TER reduces with increasing bias and temperature. In addition, the enhanced conductance at higher temperatures prevents poling the polarization state at a given voltage because the junction becomes kind of leaky, and therefore the saturated ferroelectric state is not achieved before the high current burns the junction. The reason why $J 1-2 u c$ shows more significant bias and temperature dependence than $J 2$ is related to the $\mathrm{La}_{0.5} \mathrm{Ca}_{0.5} \mathrm{MnO}_{3}$ layer which is not very insulating in its insulating state in comparison with that of the BTO layer. Therefore, junction $J 1-2 u c$ is likely to have more defect-mediated inelastic tunneling when the LCMO is at the insulating phase and more sensitive to the bias/temperature than $J 2$.

The magnetoelectric origin of the observed TER effect is supported by our measurement in high magnetic field. As shown in Fig. 9, the high magnetic field remarkably reduces the junction resistance for the high resistance state of $J 1-1 \mathrm{uc}$ $\left(12 \times 12 \mu \mathrm{m}^{2}\right)$, in which the $\mathrm{La}_{0.5} \mathrm{Ca}_{0.5} \mathrm{MnO}_{3}$ layer should be in antiferromagnetic state when polarization is pointing down. This should originate from the canting of spins in $\mathrm{La}_{0.5} \mathrm{Ca}_{0.5} \mathrm{MnO}_{3}$. On the contrary, $R-H$ curves for $J 2$ at different polarization states show nearly unchanged curvature because the doping effect from screening the ferroelectric polarization is not sufficient to change the magnetic state in the LSMO electrode.

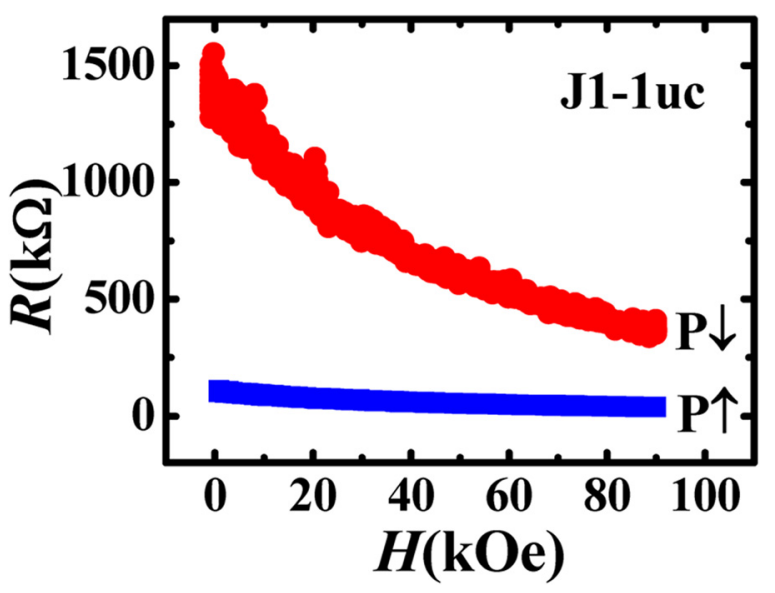

FIG. 9. Resistance vs. magnetic field up to $9 \mathrm{~T}$ at $80 \mathrm{~K}$ for $J 1-1 u c$ at different polarization states. 


\section{FERROELECTRIC CONTROL OF SPIN POLARIZATION}

Changes of the magnetic properties at the ferroelectric/ metal interface can come from the interfacial magnetoelectric coupling due to charge screening, ${ }^{36,41}$ interface bonding, ${ }^{22,42}$ or interfacial magnetocrystalline anisotropy. ${ }^{43}$ One of the significant effects predicted for MFTJ is the possible electric control of spin polarization, which is highly desirable for spintronics. We measured transport spin polarization through the TMR effect. Fig. 10 shows the TMR effect for three different samples. In $J 1-1 u c$ with a TER effect $\sim 4400 \%$, we found that the $T M R$ values are much larger in the low resistance state ( $\sim 90 \%)$ than in the high resistance state. The TMR effect is barely noticeable for the high resistance state with no sign of TMR in the negative magnetic field side and $\sim 20 \%$ on the positive field side. The irregular $R-H$ shape and the much lower $T M R$ value is also consistent with that the $\mathrm{La}_{0.5} \mathrm{Ca}_{0.5} \mathrm{MnO}_{3}$ layer becomes antiferromagnetic and therefore scatters the polarized spins (spin filter effect) in the high resistance state. The tunnel electromagnetoresistance (TEMR), the percentage ratio between the $T M R$ values for the two polarization states as defined by Garcia et al., ${ }^{17}$ is $>450 \%$ for $J 1-1 u c$. Shown in Fig. 10 (b) is sample $J 1-5 u c\left(20 \times 20 \mu \mathrm{m}^{2}\right)$ with the TER of $\sim 550 \%$, smaller than that of $J 1-1 u$ c. The TMR value is $\sim 180 \%$ at the low resistance state and $\sim 95 \%$ at high resistance state. The corresponding TEMR value is $\sim 90 \%$, five times smaller than $J 1-1 u c$. In comparison, the TEMR of $J 2$ $\left(4 \times 4 \mu \mathrm{m}^{2}\right.$ with nominally $3 \mathrm{~nm}$ BTO) without the inserted $\mathrm{La}_{0.5} \mathrm{Ca}_{0.5} \mathrm{MnO}_{3}$ layer is $\sim 35 \%$, much smaller than the MFTJs with an $\mathrm{La}_{0.5} \mathrm{Ca}_{0.5} \mathrm{MnO}_{3}$ interface layer. Since the $T M R$ value

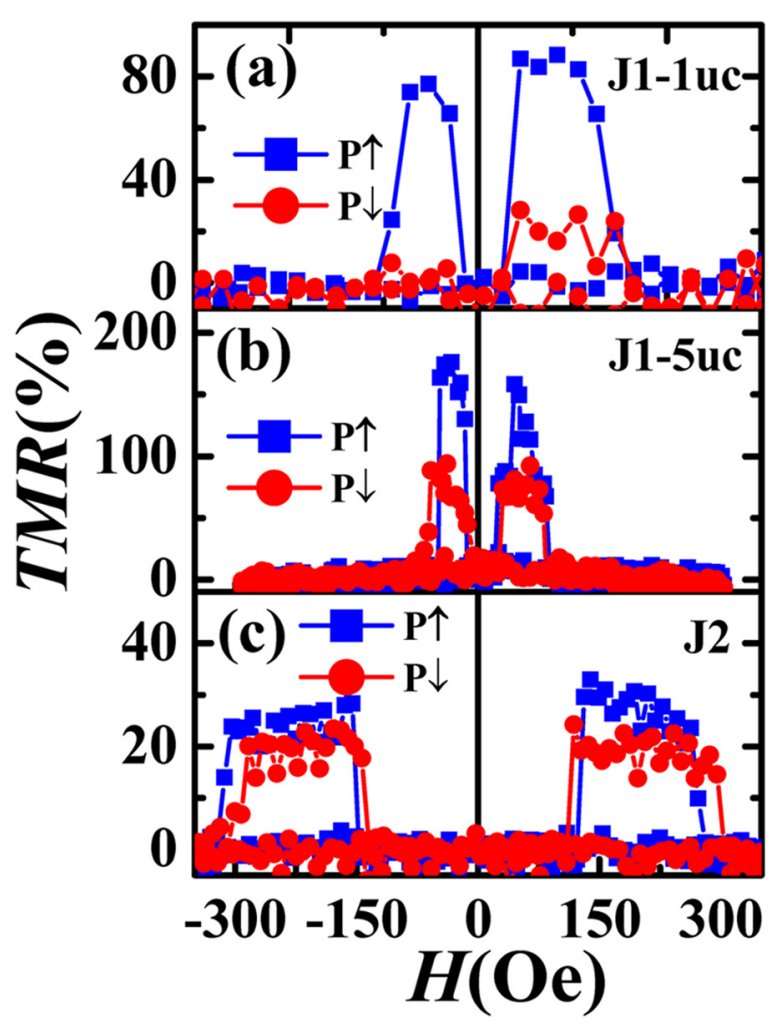

FIG. 10. Resistance vs. magnetic field scan (TMR effect) for (a) junction $J 1-1 u c$, (b) $J 1-5 u c$, and (c) $J 2$ at $80 \mathrm{~K}$. is directly proportional to the spin injection efficiency, the $T E M R$ is directly proportional to the change of spin polarization. The percentage change of spin polarization appears to be directly related to the TER value. The samples with the largest $T E R$ provide the largest change. This effect will be studied further to quantify the relationship between TER and TEMR so that the spin injection can be controlled electrically using a ferroelectric/magnetic interface.

\section{CONCLUSION}

To summarize, we have observed the four resistance state in MFTJs with sizable differences among the resistance states. Large TMR and TER effects and the resistance switching among states have been discussed in the LCMO/ $\mathrm{Ba}_{0.5} \mathrm{Sr}_{0.5} \mathrm{TiO}_{3} / \mathrm{LCMO}$ MFTJs. By selecting suitable materials, an MFTJ with four states at room temperature was achieved in LSMO/ $/ \mathrm{Ba}_{0.95} \mathrm{Sr}_{0.05} \mathrm{TiO}_{3} / \mathrm{LSMO}$ MFTJs. In order to achieve large on-off ratio of the resistance switching, we have designed a new $\mathrm{LSMO} / \mathrm{BTO} / \mathrm{La}_{0.5} \mathrm{Ca}_{0.5} \mathrm{MnO}_{3} / \mathrm{LSMO}$ MFTJ structure with a $\mathrm{La}_{0.5} \mathrm{Ca}_{0.5} \mathrm{MnO}_{3}$ layer close to ferromagnetic metal to antiferromagnetic insulator phase transition inserted in between the ferroelectric barrier and the ferromagnetic electrode. The TER effect can be enhanced by two orders of magnitude. This improvement is likely to be related to the ferroelectrically controlled ferromagnetic metallic-antiferromagnetic insulating phase transition in $\mathrm{La}_{0.5} \mathrm{Ca}_{0.5} \mathrm{MnO}_{3}$. This demonstrates the potential of MFTJ to be used in memory-onlogic applications. We have also observed that the TMR can be controlled by the ferroelectric polarization reversal, suggesting a strong electric control of spin polarization using a designed structure with proper interfaces.

\section{ACKNOWLEDGMENTS}

The work at PSU was supported in part by the DOE (Grant No. DE-FG02-08ER4653) and the NSF (Grant Nos. DMR-1207474 and DMR-1411166). The PSU NNIN Nanofabrication facilities are acknowledged. The work at USTC was supported by NSFC, NBRPC (Grant No. 2015CB921201) and FRFCU (Contract No. WK2030020026). The work at University of Nebraska-Lincoln (UNL) was supported by NSF MRSEC (Grant No. DMR-DMR-0820521). Computations were performed at the UNL Holland Computing Center. The work at ORNL was supported by the Materials Science and Engineering Division of the U.S. DOE.

${ }^{1}$ J. Ma, J. Hu, Z. Li, and C. W. Nan, Adv. Mater. 23, 1062 (2011).

${ }^{2}$ J. P. Velev, S. S. Jaswal, and E. Y. Tsymbal, Philos. Trans. R. Soc. London, Ser. A 369, 3069 (2011).

${ }^{3}$ J. A. Hutchby, R. Cavin, V. Zhirnov, J. E. Brewer, and G. Bourianoff, Computer 41, 28 (2008).

${ }^{4}$ E. Y. Tsymbal and H. Kohlstedt, Science 313, 181 (2006).

${ }^{5}$ E. Y. Tsymbal, A. Gruverman, V. Garcia, M. Bibes, and A. Barthelemy, MRS Bull. 37, 138 (2012).

${ }^{6}$ M. Julliere, Phys. Lett. A 54, 225 (1975).

${ }^{7}$ J. S. Moodera, L. R. Kinder, T. M. Wong, and R. Meservey, Phys. Rev. Lett. 74, 3273 (1995).

${ }^{8}$ S. S. P. Parkin, C. Kaiser, A. Panchula, P. M. Rice, B. Hughes, M. Samant, and S. H. Yang, Nat. Mater. 3, 862 (2004).

${ }^{9}$ V. Garcia and M. Bibes, Nat. Commun. 5, 4289 (2014).

${ }^{10}$ S. Boyn, S. Girod, V. Garcia, S. Fusil, S. Xavier, C. Deranlot, H. Yamada, C. Carretero, E. Jacquet, M. Bibes, A. Barthelemy, and J. Grollier, Appl. Phys. Lett. 104, 052909 (2014). 
${ }^{11}$ A. Chanthbouala, A. Crassous, V. Garcia, K. Bouzehouane, S. Fusil, X. Moya, J. Allibe, B. Dlubak, J. Grollier, S. Xavier, C. Deranlot, A. Moshar, R. Proksch, N. D. Mathur, M. Bibes, and A. Barthelemy, Nat. Nanotechnol. 7, 101 (2012).

${ }^{12}$ M. Y. Zhuravlev, R. F. Sabirianov, S. S. Jaswal, and E. Y. Tsymbal, Phys. Rev. Lett. 94, 246802 (2005).

${ }^{13}$ Y. P. Yao, Y. K. Liu, S. N. Dong, Y. W. Yin, S. W. Yang, and X. G. Li, Appl. Phys. Lett. 100, 193504 (2012).

${ }^{14}$ H. Kohlstedt, A. Petraru, K. Szot, A. Rudiger, P. Meuffels, H. Haselier, R. Waser, and V. Nagarajan, Appl. Phys. Lett. 92, 062907 (2008).

${ }^{15}$ Y. W. Yin, M. Raju, W. J. Hu, X. J. Weng, X. G. Li, and Q. Li, J. Appl. Phys. 109, 07D915 (2011).

${ }^{16}$ M. Hambe, A. Petraru, N. A. Pertsev, P. Munroe, V. Nagarajan, and H. Kohlstedt, Adv. Funct. Mater. 20, 2436 (2010).

${ }^{17}$ V. Garcia, M. Bibes, L. Bocher, S. Valencia, F. Kronast, A. Crassous, X. Moya, S. Enouz-Vedrenne, A. Gloter, D. Imhoff, C. Deranlot, N. D. Mathur, S. Fusil, K. Bouzehouane, and A. Barthelemy, Science 327, 1106 (2010).

${ }^{18}$ Y. K. Liu, Y. W. Yin, S. N. Dong, S. W. Yang, T. Jiang, and X. G. Li, Appl. Phys. Lett. 104, 043507 (2014).

${ }^{19}$ D. Pantel, S. Goetze, D. Hesse, and M. Alexe, Nat. Mater. 11, 289 (2012).

${ }^{20}$ Y. W. Yin, M. Raju, W. J. Hu, X. J. Weng, K. Zou, J. Zhu, X. G. Li, Z. D. Zhang, and Q. Li, Front. Phys. 7, 380 (2012).

${ }^{21}$ Z. Li, X. Guo, H. B. Lu, Z. Zhang, D. Song, S. Cheng, M. Bosman, J. Zhu, Z. Dong, and W. Zhu, Adv. Mater. 26, 7185 (2014).

${ }^{22}$ C.-G. Duan, S. Jaswal, and E. Tsymbal, Phys. Rev. Lett. 97, 047201 (2006).

${ }^{23}$ M. K. Niranjan, J. D. Burton, J. P. Velev, S. S. Jaswal, and E. Y. Tsymbal, Appl. Phys. Lett. 95, 052501 (2009).

${ }^{24}$ S. Valencia, A. Crassous, L. Bocher, V. Garcia, X. Moya, R. O. Cherifi, C. Deranlot, K. Bouzehouane, S. Fusil, A. Zobelli, A. Gloter, N. D. Mathur, A. Gaupp, R. Abrudan, F. Radu, A. Barthelemy, and M. Bibes, Nat. Mater. 10, 753 (2011).

${ }^{25}$ J. D. Burton and E. Y. Tsymbal, Phys. Rev. Lett. 106, 157203 (2011).

${ }^{26}$ J. Z. Sun and D. C. Ralph, J. Magn. Magn. Mater. 320, 1227 (2008).
${ }^{27}$ W. G. Wang, M. G. Li, S. Hageman, and C. L. Chien, Nat. Mater. 11, 64 (2012).

${ }^{28}$ J. P. Velev, C. G. Duan, J. D. Burton, A. Smogunov, M. K. Niranjan, E. Tosatti, S. S. Jaswal, and E. Y. Tsymbal, Nano Lett. 9, 427 (2009).

${ }^{29}$ Y. W. Yin, J. D. Burton, Y. M. Kim, A. Y. Borisevich, S. J. Pennycook, S. M. Yang, T. W. Noh, A. Gruverman, X. G. Li, E. Y. Tsymbal, and Q. Li, Nat. Mater. 12, 397 (2013).

${ }^{30}$ L. Jiang, W. S. Choi, H. Jeen, S. Dong, Y. Kim, M. G. Han, Y. Zhu, S. V. Kalinin, E. Dagotto, T. Egami, and H. N. Lee, Nano Lett. 13, 5837 (2013).

${ }^{31}$ M. Y. Zhuravlev, Y. Wang, S. Maekawa, and E. Y. Tsymbal, Appl. Phys. Lett. 95, 052902 (2009).

${ }^{32}$ Z. Wen, C. Li, D. Wu, A. Li, and N. Ming, Nat. Mater. 12, 617 (2013).

${ }^{33}$ D. Pantel and M. Alexe, Phys. Rev. B 82, 134105 (2010).

${ }^{34}$ D. A. Tenne, A. Bruchhausen, N. D. Lanzillotti-Kimura, A. Fainstein, R. S. Katiyar, A. Cantarero, A. Soukiassian, V. Vaithyanathan, J. H. Haeni, W. Tian, D. G. Schlom, K. J. Choi, D. M. Kim, C. B. Eom, H. P. Sun, X. Q. Pan, Y. L. Li, L. Q. Chen, Q. X. Jia, S. M. Nakhmanson, K. M. Rabe, and X. X. Xi, Science 313, 1614 (2006).

${ }^{35}$ P. Schiffer, A. P. Ramirez, W. Bao, and S. W. Cheong, Phys. Rev. Lett. 75, 3336 (1995).

${ }^{36}$ J. Burton and E. Tsymbal, Phys. Rev. B 80, 174406 (2009).

${ }^{37}$ W. F. Brinkman, R. C. Dynes, and J. M. Rowell, J. Appl. Phys. 41, 1915 (1970).

${ }^{38}$ J. Z. Sun, L. Krusin-Elbaum, P. R. Duncombe, A. Gupta, and R. B. Laibowitz, Appl. Phys. Lett. 70, 1769 (1997).

${ }^{39}$ Y. Lu, M. Tran, H. Jaffrès, P. Seneor, C. Deranlot, F. Petroff, J. M. George, B. Lépine, S. Ababou, and G. Jézéquel, Phys. Rev. Lett. 102, 176801 (2009).

${ }^{40}$ L. I. Glazman and K. A. Matveev, Sov. Phys. JETP 67, 1276 (1988).

${ }^{41}$ C. A. F. Vaz, J. Hoffman, Y. Segal, J. W. Reiner, R. D. Grober, Z. Zhang, C. H. Ahn, and F. J. Walker, Phys. Rev. Lett. 104, 127202 (2010).

${ }^{42}$ J. Lee, N. Sai, T. Cai, Q. Niu, and A. A. Demkov, Phys. Rev. B 81, 144425 (2010).

${ }^{43}$ C. G. Duan, J. P. Velev, R. F. Sabirianov, W. N. Mei, S. S. Jaswal, and E. Y. Tsymbal, Appl. Phys. Lett. 92, 122905 (2008). 